\title{
Review Article \\ Theory of Mind after Severe Acquired Brain Injury: Clues for Interpretation
}

\author{
U. Bivona $\mathbb{D}^{1},{ }^{1}$ R. Formisano, ${ }^{1}$ L. Mastrilli, ${ }^{1}$ S. Zabberoni $\mathbb{D},{ }^{1,2}$ \\ C. Caltagirone $\mathbb{D}^{1,3}$ and A. Costa ${ }^{1,2}$ \\ ${ }^{1}$ Fondazione Santa Lucia, IRCCS, Rome, Italy \\ ${ }^{2}$ Università telematica Niccolò Cusano, Rome, Italy \\ ${ }^{3}$ Università Tor Vergata, Rome, Italy \\ Correspondence should be addressed to U. Bivona; u.bivona@hsantalucia.it
}

Received 23 January 2018; Revised 24 April 2018; Accepted 4 June 2018; Published 5 July 2018

Academic Editor: Diane Ruge

Copyright (C) 2018 U. Bivona et al. This is an open access article distributed under the Creative Commons Attribution License, which permits unrestricted use, distribution, and reproduction in any medium, provided the original work is properly cited.

\begin{abstract}
Background. Recently, increased interest has been shown in Theory of Mind (ToM) abilities of individuals with severe acquired brain injury (sABI). ToM impairment following sABI can be associated with altered executive functioning and/or with difficulty in decoding and elaborating emotions. Two main theoretical models have been proposed to explain the mechanisms underlying ToM in the general population: Theory Theory and Simulation Theory. This review presents and discusses the literature on ToM abilities in individuals with sABI by examining whether they sustain the applicability of the Theory Theory and/or Simulation Theory to account for ToM deficits in this clinical population. We found 32 papers that are directly aimed at investigating ToM in sABI. Results did not show the univocal predominance of one model with respect to the other in explaining ToM deficits in sABI. We hypothesised that ToM processes could be explained by coinvolvement of the two models, i.e., according to personal experience, cognitive features, or the emotional resources of the persons with sABI.
\end{abstract}

\section{General Introduction}

Theory of Mind (ToM) is the ability to infer others' intentions and beliefs [1-6]. It is crucial for sustaining social cognition, which is a prerequisite for adaptive learning and psychological satisfaction of the human being.

Two main theoretical models have been proposed to explain ToM processes. The first model is Theory Theory [712], according to which a human being infers others' intentions and beliefs by acquiring and deploying something that is quite similar to a scientific theory. Theory theorists posit that people apply general principles (e.g., rules shared within their own culture and tacitly known causal laws) regarding social relationship in order to make hypotheses about others' mental reasoning with specific involvement of executive functions, abstract reasoning, and working memory $[12,13]$.

In contrast to the Theory Theory, the Simulation Theory [10] sees ToM abilities as a result of a simulation process based on the autobiographical experience of an individual that allows "putting himself in the other's shoes". Within this framework, the individual remodels the other's experience on himself to make predictions about the other's behaviour, and emotional-affective elaboration is supposed to play an important role. This model emerged following the discovery of mirror neurons $[14,15]$ which can be considered an important phylogenetic or ontogenetic precursor of the ability to assume the other's perspective. However, according to the same authors $[14,16]$, the mirror neuron system is not in itself able to explain the ability to ascribe propositional attitudes such as beliefs.

Several studies document that ToM may be impaired in adult individuals with psychiatric and neurological diseases (see [17] for a review); furthermore, in recent decades growing interest has been shown in investigating ToM abilities also in persons with severe acquired brain injury (sABI). Results consistently document that, compared to healthy controls, patients with sABI perform worse on ToM tasks [1830]. 


\section{ToM Functioning in Individuals with sABI}

As mentioned above, ToM was found to be impaired in patients with $\mathrm{SABI}$ in association with altered executive functions [31-33] and/or difficulty in decoding and elaborating emotions [34-37].

It has also been recently demonstrated that ToM impairment in patients with sABI may be associated with decreased quality of life of their caregivers [29]. In this regard, note that ToM impairments can be viewed within a more general biopsycho-social framework in which the severity of symptoms often causes poor family, social, and work re-entry outcomes.

According to the brain lesion site, dimension, and depth, patients with sABI may present with several cognitiveaffective changes. Memory, attention, and executive systems are mainly involved $[32,33,51]$. Impulsivity and disinhibition are also frequently observed $[37,55,61]$. At the end of the 1970 s, it was pointed out that social cognition impairment is one of the most severe behavioural consequences of an sTBI [62]. Subsequent studies confirmed that ToM can be severely affected after an sABI [18, 19, 21-24, 27-30, 63]. In particular, in individuals with severe TBI difficulty in identifying the source of an interpersonal conflict or the meaning of social behaviours has been documented [40, 64-66]. The findings of other studies were similar when patients were required to interpret nonverbal social interactions $[67,68]$ or to assume the perspective of a specific character in a story ([19]; see also [63], for an exhaustive review of this topic). Indeed, these patients may exhibit important changes in personality features that result in low sensitivity to others' needs [29, 69], poor interest and childishness [18, 70], and egocentrism [71].

Taken together these changes significantly increase the burden and psychophysical distress of caregivers [29, 72-74] and negatively influence patients' therapeutic outcomes [18, 73]. In fact, results of different studies document that quality of life of both patients [75] and their caregivers [29, 72-74, 76] can be significantly affected.

ToM deficits should also be viewed within the general neuropsychological profile of the patient with sABI. Indeed, in some studies [22, 33, 40,44, 51, 65, 77], a positive correlation was found between working memory, processing speed, inhibition and the ability to be flexible, and patient's performance on ToM tasks. However, results of other studies document a possible dissociation between cognitive impairment after sABI and social cognition deficits [67, 78-80]. In this regard, some authors hypothesised that ToM and other cognitive domains should be considered as independent cognitive systems $[44,51,54]$.

Particular attention should also be given to impaired self-awareness (ISA) after sABI, considered as the ability to be aware of one's own thoughts, feelings, and mental states [81], as well as "the capacity to perceive the self in relatively objective terms whilst maintaining a sense of subjectivity" [82]. Indeed, self-awareness is frequently impaired after an sABI [83-85] and is characterised by partially or totally reduced ability to recognise problems caused by damaged brain function. In a recent study of patients with sTBI and healthy controls, we demonstrated a significant relationship between ISA and perspective-taking difficulty [28], which could account for patients' difficulty in managing social relationships. In another study poor performance of patients with sTBI on ToM performance-based tasks was also found to be correlated with poor quality of life reported by their caregivers [29], which was assessed by the QOLIBRI questionnaire $[86,87]$. This latter finding supports the clinical observation that caregivers may be upset by the poor sensitivity exhibited by patients with respect to the potential consequences of their behaviours (i.e., agitation, irritability, aggressiveness, apathy, and disinhibition; see [88] for more details) on the persons who care for them daily.

To summarize, available data document that ToM abilities may be reduced after sABI and that this impairment may also affect caregivers' quality of life.

\section{Aims of the Review}

Theory Theory and Simulation Theory models lead to different hypotheses about the processes implied in ToM functioning. However, the applicability and predictive value of the two models to account for ToM impairment following sABI must still be clarified.

Therefore, in this review, we present and discuss published data on ToM abilities in individuals with sABI by examining whether they sustain the applicability of the Theory Theory or Simulation Theory in accounting for ToM deficits in this clinical population.

\section{Methods}

This review was carried out using the research databases PubMed, PsycINFO, and Scopus to identify coherent studies on the topic investigated here up until April 2018. The literature search was completed by combining four keywords related to brain damage on one side and seven keywords related to social cognition ability on the other, as reported in Table 1. We also reviewed the reference lists of previously published reviews and all original studies to identify all relevant papers for inclusion.

All papers considered eligible for the study were then included in the review process if they met all of the following inclusion criteria: (1) they included patients with sABI, (2) examined ToM abilities, (3) included a healthy control group, and (4) were written in English.

After exclusion of each overlap between these three databases, only 32 papers met the above inclusion criteria. In particular, they represented 7.2\% of the total number (445) of papers found eligible on PubMed, $14.5 \%$ of the total papers (220) on PsycINFO, and 9.6\% of the total papers (332) on Scopus.

4.1. Theory Theory and $s A B I$. As mentioned above, Theory Theory underlines the role of cognitive processes (i.e., logical reasoning, working memory, executive functioning, and pragmatic language) in understanding others' mental states. Therefore, it can be hypothesised that the finding of a significant association between deficits of cognitive-executive 
TABLE 1: Search results.

\begin{tabular}{|c|c|c|c|c|}
\hline 1st keyword & 2nd keyword & PubMed results & PsycINFO results & Scopus results \\
\hline \multirow{7}{*}{ Severe acquired brain injury } & social cognition & 188 & 27 & 34 \\
\hline & theory of mind & 41 & 1 & 7 \\
\hline & mentalizing & 41 & 13 & 1 \\
\hline & attributions & 9 & 2 & 7 \\
\hline & perspective taking & 7 & 4 & 2 \\
\hline & Theory Theory & 135 & 1 & 26 \\
\hline & Simulation Theory & 3 & 1 & 1 \\
\hline \multirow{7}{*}{ Craniocerebral trauma } & social cognition & 608 & 0 & 30 \\
\hline & theory of mind & 88 & 1 & 3 \\
\hline & mentalizing & 93 & 0 & 0 \\
\hline & attributions & 31 & 0 & 16 \\
\hline & perspective taking & 25 & 0 & 2 \\
\hline & Theory Theory & 719 & 0 & 84 \\
\hline & Simulation Theory & 15 & 0 & 10 \\
\hline \multirow{7}{*}{ Head injury } & social cognition & 633 & 14 & 217 \\
\hline & theory of mind & 92 & 8 & 24 \\
\hline & mentalizing & 97 & 1 & 3 \\
\hline & attributions & 34 & 16 & 145 \\
\hline & perspective taking & 27 & 5 & 10 \\
\hline & Theory Theory & 815 & 2 & 433 \\
\hline & Simulation Theory & 19 & 1 & 31 \\
\hline \multirow{7}{*}{ Severe traumatic brain injury } & social cognition & 460 & 117 & 544 \\
\hline & theory of mind & 69 & 82 & 80 \\
\hline & mentalizing & 69 & 8 & 7 \\
\hline & attributions & 14 & 10 & 210 \\
\hline & perspective taking & 16 & 11 & 16 \\
\hline & Theory Theory & 398 & 12 & 470 \\
\hline & Simulation Theory & 11 & 0 & 14 \\
\hline \multicolumn{2}{|c|}{ Total number of items after exclusion of overlapping papers } & 445 & 220 & 332 \\
\hline
\end{tabular}

abilities and ToM impairments supports the applicability of the Theory Theory framework in this clinical population.

In this vein, Apperly and coll. [32] administered the false belief paradigm $[27,32,89]$ to patients with sABI and found that poor ToM performance was significantly correlated with poor performance on executive tests. In patients with TBI, Henry and coll. [33] documented a significant association between performance on verbal fluency [90], i.e., a test that involves some aspects of executive functioning [91], and the Reading the Mind in the Eyes test [58]. Results of other studies that used the Faux Pas paradigm [57] seem to confirm the above association between executive and ToM functioning in individuals with sTBI $[28,51]$ (see Table 2).

Other investigations indirectly indicate an association between executive functioning and social cognition in sABI patients. Indeed, Channon and coll. [41] revealed a significant positive correlation between the ability to mentalize and the comprehension of sarcasm, which according to the authors could be the result of low abstract reasoning ability [41]. Moreover, by administering the Social Problem Resolution Task and the Social Problem Fluency Task [59], Channon and Crawford [40] demonstrated worse performance in patients with $\mathrm{ABI}$ than in healthy controls; this suggests that there may be a significant association between ToM and the ability to implement strategic social skills. More recently, McDonald et al. [49] documented a significant association between poorer social cognition and difficulty in assessing the sincerity of a speaker in patients with severe TBI.

A more specific association between working memory and ToM functioning was documented in a series of studies. Bibby and McDonald [22] administered individuals with sTBI verbal (first-order and second-order) [57] and nonverbal (cartoon) ToM tasks $[22,60]$ and verbal and nonverbal tasks requiring them to make general inferences. These authors found a significant association between ToM functioning and performance on tasks requiring working memory and implicit language. Honan and coll. [45] investigated whether performance on ToM tasks (i.e., different versions of a ToM procedure that varied for kind of executive demands required) depends on executive impairment in patients with severe TBI. Results were not univocal; they show that TBI patients' performance tended to be worse than that of $\mathrm{HCs}$ $(p=0.053)$ in a low executive demand condition. However, the findings of this study also document a specific association 


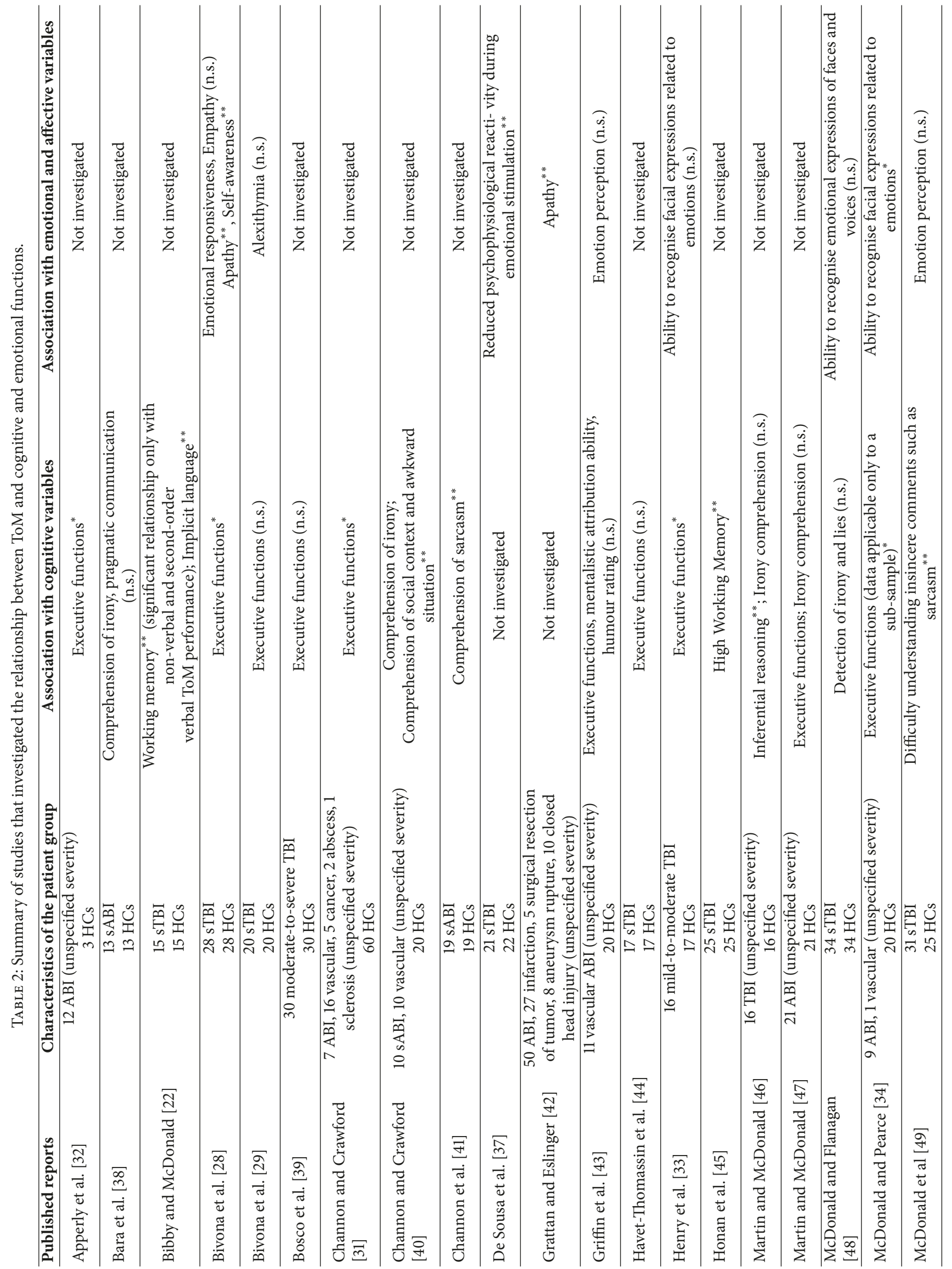




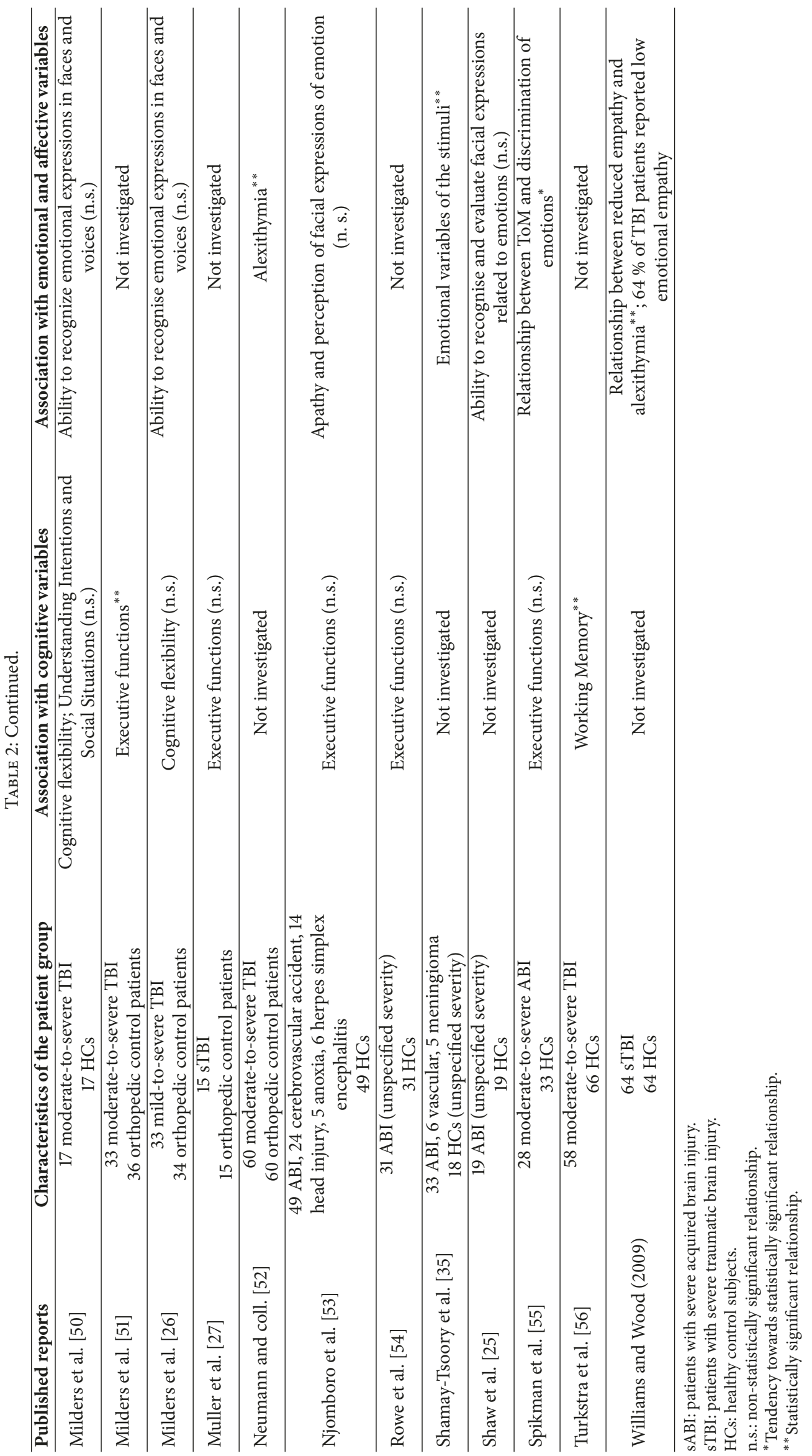


between ToM performance and working memory processes (but not with flexibility and inhibition processes) as revealed by poorer ToM performance of sTBI patients than HCs in a condition with high demands on working memory processes. The association between working memory processes and ToM abilities in patients with sTBI and healthy controls was also investigated in a more recent study [56] by administering a ToM procedure (i.e., the Video Social Inference Test) in which the working memory load was manipulated. Results of this study showed that patients with sTBI performed worse than HCs on the ToM task; moreover, an association between working memory load and ToM performance was found in both experimental groups. However, by directly testing the effect of working memory load on the between group difference in ToM performance, the authors found that the reduced efficiency of sTBI patients' ToM processes could not be fully accounted for by their working memory abilities.

Taken together, these findings support the applicability of the predictions of the Theory Theory model to explain ToM dysfunctions in sABI because they document a significant association between performance on ToM tasks and on tests sensitive to executive functioning. However, other studies do not sustain such a relationship. In fact, Havet-Thomassin et al. [44] administered the Reading the Mind in the Eyes' test [58] and the Character Intention Task [44] to patients with sTBI and healthy controls and found no significant correlations between ToM indexes and executive performance (measured by administering the Tower of London planning task [92], the Stroop Colour Word Test [93], the Modified Card Sorting Test [94], and the Trail Making Test [95]). Similarly, other studies $[27,39,54]$ found no significant association between ToM performance and various measures of executive functioning. In this vein, it should also be noted that, as discussed above, Turkstra et al. [56] showed that the ToM performance of patients with sTBI could not be fully accounted for by the involvement of working memory weakness.

In synthesis, the evidence reported above shows a heterogeneous picture that does not allow drawing firm conclusions about the involvement of executive processes in ToM functioning in patients with sABI.

4.2. Simulation Theory and sABI. As stated above, the Simulation Theory primarily posits that humans are able to "shape on the self" others' experience [14]. The ability to simulate and decode emotional experience plays a crucial role in this model with respect to pure cognitive skills. In this vein, it could be argued that emotional disturbances due to sABI are strictly related to ToM deficits in these patients. Indeed, some disturbances, such as an exaggeration or a reduction of emotional-behavioural activity, often occur after severe brain lesions. These disturbances may cause inappropriate social behaviour, impulsivity, emotional lability, irritability, loss of self-control, emotional flattening, and difficulty in recognising others' emotions and facial expressions $[37,55]$. Accordingly, a significant association between emotional changes and ToM deficits could, at least theoretically, indicate the validity of the Simulation Theory model.

In line with this perspective, apathy was reported to be associated with poor ToM in patients with ABI [28,
42]. Moreover, Williams and Wood [36] found a significant relationship between empathic abilities (assessed by the $\mathrm{Bal}$ anced Emotional Empathy Scale, BEES, [96]) and alexithymia (assessed by the Toronto Alexithymia Scale, TAS-20, [97]). It should also be noted that in this study more than $64 \%$ of the patients involved showed a total loss, or a significant reduction, of their empathic abilities. Moreover, Neumann and coll. [52] found that patients with moderate-to-severe TBI who showed external-oriented thinking (i.e., a tendency to avoid reasoning on (about) their own emotions) were more likely to exhibit difficulty in recognising others' emotions as well as assuming others' perspectives. De Sousa et al. [37] also documented in patients with sABI that failure to assume others' emotional perspectives was significantly associated with reduced physiological response to facial expressions with emotional valence; this suggests that there is a significant relationship between empathic abilities and capacity to code emotions (see Table 2).

In line with the above findings, in a previously mentioned study, we documented that in patients with severe TBI poor self-awareness was associated with poor performance on Faux Pas tests [28]. This finding could indicate that patients' reduced self-awareness (i.e., poor ability "to put themselves in their own shoes") is related to their poor ability to take the other's perspective (i.e., poor ability in "putting themselves in the other's shoes").

Taken together, the results of the above-cited studies seem to indicate that emotional processing, self-thinking, and ToM abilities are significantly interrelated; thus indirectly supports the hypotheses and predictions of the Simulation Theory.

However, Njomboro et al. [53] reported results that are not in line with the above conclusion, as they failed to find a significant association between affective disorders (i.e., apathy) and ToM functioning (assessed with false belief tasks) in patients with severe ABI. In particular, the results of this study document that patients with apathy do not differ from those without apathy in performing ToM tasks [53].

In summary, although in line with the Simulation Theory some findings document a significant association between ToM performance and emotional/affective variables, others (see Table 2 for details) did not show clear evidence of this association. Therefore, no firm conclusions can be made in sABI about the involvement of emotional/affective disorders in ToM functioning.

\section{Are Theory Theory and Simulation Theory Both Involved in Explaining ToM Impairments after sABI?}

The present review aimed to determine the potential applicability of both Theory Theory and Simulation Theory to account for the pattern of ToM deficits observed in the field of sABI. We do not, however, believe that the studies taken into account allow drawing firm conclusions about the superiority of one model over the other.

According to the Theory Theory, cognitive and, in particular, executive functions play a critical role in mediating ToM processes. Based on this assumption, we should expect to 
find a strong association between performance on ToM tasks and on tests investigating executive functioning in patients with sABI. Although the findings of some studies are in line with this hypothesis, other investigations failed to find an association ([22, 26, 27, 38, 43, 47, 48, 50, 54, 55]).

According to the Simulation Theory, instead, ToM capacities depend on the ability to simulate others' experience [10]. Therefore, the ability to code and process emotional experience by using our own mind to simulate the target's mental processes should be mainly involved in this case. Indeed, the literature examined here provides some support for the applicability of this model, in particular when low selfawareness and emotional disturbances were associated with poor ToM performance. However, also in this case, findings do not seem to be univocal $[25,26,28,29,43,46,48,50]$ (see Table 2).

The above observations could suggest that patients with sABI fail to take the other's perspective because of their reduced cognitive and/or emotional resources. Indeed, in these patients cognitive (linked to Theory Theory) and emotional/affective (linked to Simulation Theory) functions can be contemporarily or differentially involved. This evidence could support the view that the two theoretical models are not mutually exclusive in explaining ToM disorders in these individuals. Indeed, a patient with prevalent cognitive impairment can show ToM deficits due to difficulty in applying purely abstract logical reasoning, which hampers the ability to take into account the different elements of the presented situation. Conversely, a patient with a predominant emotional disorder can fail to take the other's perspective mainly because of difficulty implementing simulating mechanisms.

The evidence presented in this review does not allow documenting a clear dissociation between the Theory Theory and Simulation Theory models in sABI, but provides some clues that help explain ToM functioning also in the healthy population. In this regard, it may be useful to report the classic Tees/Crane experiment [98]. In this experiment, subjects are asked to answer a question related to the short story of Mr Crane and Mr Tees, who are scheduled to depart from the airport on different flights at the same time. Mr Crane and $\mathrm{Mr}$ Tees go to the airport in the same car, get caught in traffic, and arrive at the airport with a 30-minute delay with respect to their scheduled departure times. Mr Crane is told that his flight left on time and Mr Tees is told that his flight was delayed and left just five minutes before his arrival at the airport. The subjects are required to answer the question: "Who is likely to be more upset?". The experiment showed that $96 \%$ of the subjects thought that Mr Tees would be more upset. It can be hypothesised that in order to answer the experimental questions subjects can rely on both affective (i.e., referring to the Simulation Theory) and cognitive (i.e., referring to the Theory Theory) processes. According to the Simulation Theory subjects answer by simulating the situation, i.e., by using their own mental state to predict how the two characters in the story feel. In fact, the subjects can take the characters' perspectives by mentally reexperiencing similar situations in their past. However, according to the Theory Theory model, different mechanisms might be involved in subjects who have never had a similar experience. In this case, individuals might apply purely abstract reasoning that would allow taking into account the different elements of the described situation.

Future studies are needed to better clarify the hypothesis of a coinvolvement of Theory Theory and Simulation Theory assumptions in explaining ToM functioning.

\section{Sampling and Measurement}

As discussed above, we cannot draw firm conclusions about the superiority of one model with respect to the other. Indeed, some methodological limitations make it impossible to clearly understand the mechanisms underlying ToM functioning in patients with sABI.

First, the heterogeneity of samples across studies, especially those conducted on sTBI, is the first critical question. Indeed, many studies did not take into account the severity of the brain injury (i.e., mild, moderate, or severe, according to the Glasgow Coma Scale score [99]), which differentiates patients in terms of their cognitive, emotional, and behavioural functioning. Indeed, including patients with different levels of ABI severity in the same statistical analysis represents a significant bias; thus, caution is required in interpreting the results. Sample heterogeneity also makes it difficult to directly compare the results of different studies.

Another important point is the evidence that the available data are correlational in nature. This makes it difficult to examine the association between cognitive and/or emotional factors and ToM in causal terms. In this regard, no studies have investigated the possible effect on ToM ability of specific rehabilitation training for persons with sABI that is focused on the empowerment of emotional-cognitive functioning. In fact, future studies could investigate whether enhancing executive functions in patients with sABI might improve ToM in order to determine whether executive functions are involved in ToM processes in these patients.

Another critical point refers to the underestimation of poor self-awareness after sABI [28, 88, 100-102]. As stated above, individuals with reduced self-awareness can partially or totally neglect the existence of deficits related to brain injury and, consequently, they cannot accurately recognise functional consequences, including those referring to emotional, personality, and social competency changes. Moreover, it should be noted that the recovery of self-awareness is conceived as a multilevel hierarchical process [103] that starts at an intellectual level (patients only cognitively recognise the existence of a deficit related to brain injury) and passes through an emergent level (patients recognise a deficit when they are faced with it) to finally reach an anticipatory level of self-awareness (i.e., patients are able to plan their activities on the basis of their actual condition and residual deficits). According to this model, even when patients become intellectually self-aware of their deficits it might still be difficult for them to recognise emotional and ToM difficulties that occur in social relationships, as well as to prevent them only on the basis of their intellectual self-awareness.

The patients' difficulties in monitoring and describing their own condition can also affect their ability to reliably self-report ToM competences. Nevertheless, most of the studies that administered self-report ToM measures did not 


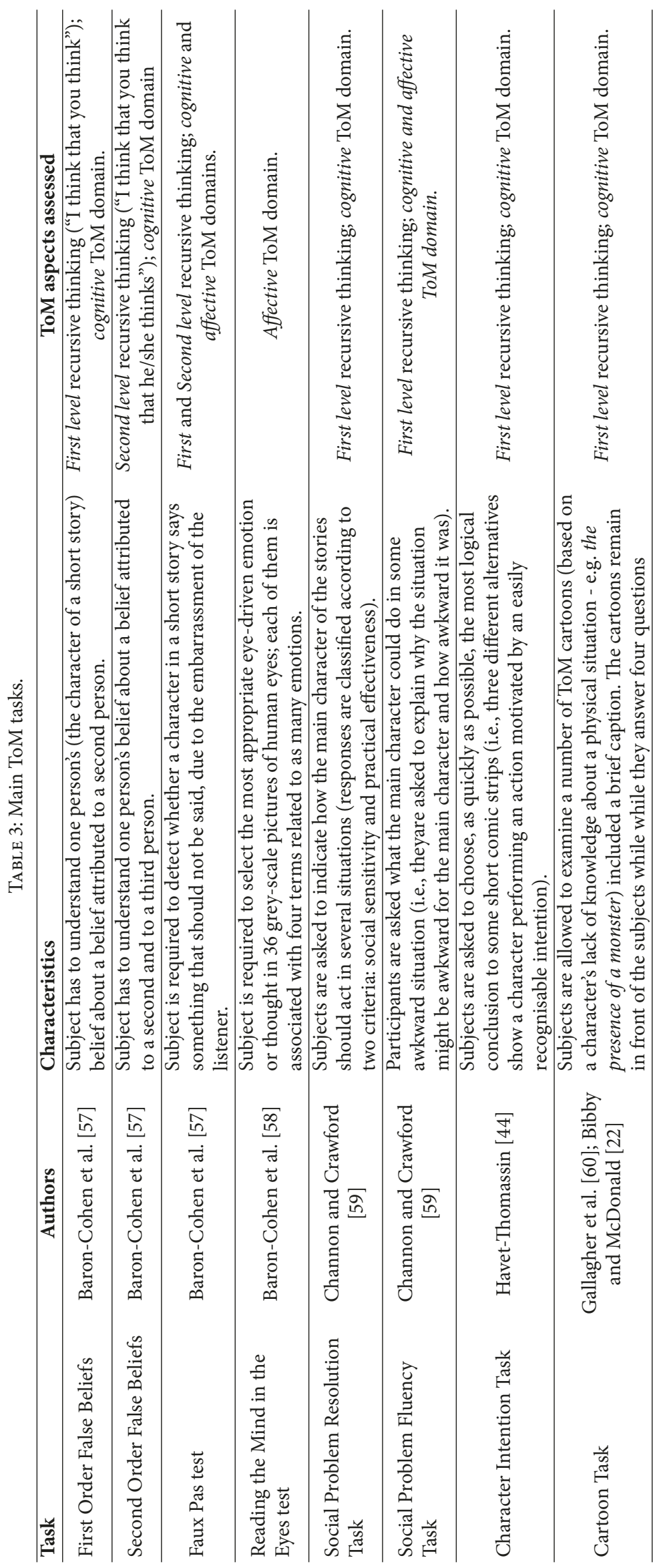


control for self-awareness disorders. Indeed, it might be paradoxical to investigate ToM problems in an individual who is unaware of the problem using explicit questions about that problem [28]. Impaired self-awareness together with cognitive deficits (mnesic and attentional in particular) could make it difficult to recall the situations cited in most selfreport questionnaires (e.g., "I really get involved with the feelings of the characters in a novel" [104]) and to remember how the patient usually feels in those situations. Therefore, since individuals may provide potentially unreliable answers because of their reduced self-awareness, the selective use of self-report questionnaires to assess perspective taking in patients with sABI should be avoided [27, 28, 36, 96, 104, 105].

To surmount the above limitations, performance-based tools (see Table 3 for details) that assess patients' ability to put themselves in the shoes of the character of a story (described verbally or by pictures) and to make decisions "as if they were that character" are recommended. Indeed, these tasks require subjects to "objectively" judge what they observe during the task, not to subjectively describe their inner states. In fact, in the above-cited study [28], which investigated the possible difference between a self-report and a performance-based tool in detecting social cognition in patients with sTBI and different levels of self-awareness, it was demonstrated that only the hierarchically more complex performance-based tools (i.e., Faux Pas tests) were able to clearly discriminate between patients with poor self-awareness and patients with adequate self-awareness and healthy controls.

However, it should be noted that to date no psychometric tool has been proposed as a "gold standard" in terms of validity and reliability in assessing perspective-taking abilities after sABI. Basically, the different psychometric characteristics of the instruments utilised strongly limit the possibility of comparing results between studies. Further studies are needed to better address this issue and, in particular, to verify our suggestions regarding the usefulness of using measures to assess perspective-taking abilities in patients with sABI only after controlling for their level of self-awareness.

\section{Conclusions}

Impaired social cognition may severely affect the quality of life of persons with sABI and their caregivers [29]. Therefore, in this population understanding the mechanisms involved in ToM impairment is an important clinical issue. This review aimed to examine the applicability of Theory Theory and Simulation Theory models to explain ToM impairments in patients with sABI. These two models are based on different theoretical assumptions: according to the former, mentalizing should mainly involve cognitive strategies; conversely, the second model points out the role of emotional and simulation processes in assuming the other's perspective.

The present review highlights the difficulty of drawing firm conclusions about the applicability of the two models in the field of sABI. In fact, results from several studies show a significant association between executive functions and ToM, in line with the Theory Theory model, whereas other findings document a significant association between ToM and emotional/affective variables, congruently with the assumptions of the Simulation Theory. We interpreted the presence of studies in line with each of the proposed theoretical frameworks as potential evidence of the coinvolvement of mechanisms posited by both of these models in accounting for ToM impairments in sABI. In fact, both cognitive and emotional/affective disorders can affect the ToM abilities of these patients.

Further studies, possibly involving behavioural, neurophysiological, and neuroimaging techniques, are needed to better understand the nature of the processes underlying social cognition and, in particular, ToM functioning after sABI. Indeed, gaining knowledge about ToM functional characteristics in this clinical population could lead to the possibility of providing ad hoc rehabilitative interventions, useful not only to the patients but also to their caregiving system. In this regard, an early neuropsychological assessment of both cognitive and emotional post-sABI deficits could help rehabilitation teams treat ToM deficits better by choosing the most suitable approach based on the most available (cognitive and/or emotional) resources.

Finally, it is important that in the future researchers pay specific attention to some clinical aspects that can affect the interpretation of results. In particular, we refer to (a) the aetiology and severity of brain injury, (b) the neuropsychological and neuropsychiatric features of the patients, and (c) their level of self-awareness. All of these aspects should be taken into account in order to have homogeneous samples and more reliable findings.

\section{Conflicts of Interest}

The authors declare that they have no conflicts of interest regarding the publication of this paper.

\section{References}

[1] S. Baron-Cohen, A. M. Leslie, and U. Frith, "Does the autistic child have a "theory of mind"?" Cognition, vol. 21, no. 1, pp. 3746, 1985.

[2] L. Brothers and B. Ring, "A neuroethological framework for the representation of minds," Cognitive Neuroscience, vol. 4, no. 2, p. $-118,1992$.

[3] C. D. Frith and U. Frith, "Interacting minds-a biological basis," Science, vol. 286, no. 5445, pp. 1692-1695, 1999.

[4] U. Frith and C. D. Frith, "Development and neurophysiology of mentalizing," Philosophical Transactions of the Royal Society B: Biological Sciences, vol. 358, no. 1431, pp. 459-473, 2003.

[5] M. Spinella, "Prefrontal substrates of empathy: Psychometric evidence in a community sample," Biological Psychology, vol. 70, no. 3, pp. 175-181, 2005.

[6] C. D. Frith and U. Frith, "The neural basis of mentalizing," Neuron, vol. 50, no. 4, pp. 531-534, 2006.

[7] S. Stich and S. Nichols, "Folk Psychology: Simulation or Tacit Theory?" Mind and Language, vol. 7, no. 1-2, pp. 35-71, 1992.

[8] A. Gopnik and H. M. Wellman, "Why the child's theory of mind really is a theory," Mind and Language, vol. 7, no. 1-2, pp. 145-171, 1992.

[9] A. Gopnik and A. Meltzoff, Words, Thoughts and Theories, MIT Press, Cambridge, UK, 1997. 
[10] M. Davies and T. Stone, Folk Psychology: The Theory of Mind Debate, Blackwell, 1995.

[11] M. Davies and T. Stone, "Folk Psychology and Mental Simulation," Royal Institute of Philosophy Supplement, vol. 43, pp. 5382, 1998.

[12] P. Carruthers and P. K. Smith, Theories of Theories of Mind, vol. 38, Cambridge University Press, Cambridge, UK, 1996.

[13] P. M. Churchland, Folk Psychology and the Explanation of Human Behaviour, Greenwood JD, 1991.

[14] V. Gallese and A. Goldman, "Mirror neurons and the simulation theory of mind-reading," Trends in Cognitive Sciences, vol. 2, no. 12, pp. 493-501, 1998.

[15] V. Gallese, C. Keysers, and G. Rizzolatti, "A unifying view of the basis of social cognition," Trends in Cognitive Sciences, vol. 8, no. 9, pp. 396-403, 2004.

[16] J. Decety and J. Grèzes, “The power of simulation: Imagining one's own and other's behavior," Brain Research, vol. 1079, no. 1, pp. 4-14, 2006.

[17] A. Ibáñez, A. M. García, S. Esteves et al., "Social neuroscience: undoing the schism between neurology and psychiatry," Social Neuroscience, vol. 13, no. 1, pp. 1-39, 2018.

[18] L. Elsass and G. Kinsella, "Social interaction following severe closed head injury," Psychological Medicine, vol. 17, no. 1, pp. 6778, 1987.

[19] M. V. Spiers, J. A. Pouk, and J. M. Santoro, "Examining perspective-taking in the severely head-injured," Brain Injury, vol. 8, no. 5, pp. 463-473, 1994.

[20] E. Winner, H. Brownell, F. Happé, A. Blum, and D. Pincus, "Distinguishing lies from jokes: Theory of mind deficits and discourse interpretation in right hemisphere brain-damaged patients," Brain and Language, vol. 62, no. 1, pp. 89-106, 1998.

[21] L. S. Turkstra, T. M. Dixon, and K. K. Baker, "Theory of Mind and social beliefs in adolescents with traumatic brain injury," NeuroRehabilitation, vol. 19, no. 3, pp. 245-256, 2004.

[22] H. Bibby and S. McDonald, "Theory of mind after traumatic brain injury," Neuropsychologia, vol. 43, no. 1, pp. 99-114, 2005.

[23] M. Schulte-Rüther, H. J. Markowitsch, G. R. Fink, and M. Piefke, "Mirror neuron and theory of mind mechanisms involved in face-to-face interactions: A functional magnetic resonance imaging approach to empathy," Cognitive Neuroscience, vol. 19, no. 8, pp. 1354-1372, 2007.

[24] S. G. Shamay-Tsoory and J. Aharon-Peretz, "Dissociable prefrontal networks for cognitive and affective theory of mind: A lesion study," Neuropsychologia, vol. 45, no. 13, pp. 3054-3067, 2007.

[25] P. Shaw, E. Lawrence, J. Bramham, B. Brierley, C. Radbourne, and A. S. David, "A prospective study of the effects of anterior temporal lobectomy on emotion recognition and theory of mind," Neuropsychologia, vol. 45, no. 12, pp. 2783-2790, 2007.

[26] M. Milders, M. Ietswaart, J. R. Crawford, and D. Currie, "Social behavior following traumatic brain injury and its association with emotion recognition, understanding of intentions, and cognitive flexibility," Journal of the International Neuropsychological Society, vol. 14, no. 2, pp. 318-326, 2008.

[27] F. Muller, A. Simion, E. Reviriego et al., "Exploring theory of mind after severe traumatic brain injury," Cortex, vol. 46, no. 9, pp. 1088-1099, 2010.

[28] U. Bivona, A. Riccio, P. Ciurli et al., "Low self-awareness of individuals with severe traumatic brain injury can lead to reduced ability to take another person's perspective," The Journal of Head Trauma Rehabilitation, vol. 29, no. 2, pp. 157-171, 2014.
[29] U. Bivona, R. Formisano, S. De Laurentiis et al., "Theory of mind impairment after severe traumatic brain injury and its relationship with caregivers' quality of life," Restorative Neurology and Neuroscience, vol. 33, no. 3, pp. 335-345, 2015.

[30] S. McDonald, A. Gowland, R. Randall, A. Fisher, K. OsborneCrowley, and C. Honan, "Cognitive factors underpinning poor expressive communication skills after traumatic brain injury: Theory of mind or executive function?" Neuropsychology, vol. 28, no. 5, pp. 801-811, 2014.

[31] S. Channon and S. Crawford, "The effects of anterior lesions on performance on a story comprehension test: Left anterior impairment on a theory of mind-type task," Neuropsychologia, vol. 38, no. 7, pp. 1006-1017, 2000.

[32] I. A. Apperly, D. Samson, C. Chiavarino, and G. W. Humphreys, "Frontal and temporo-parietal lobe contributions to theory of mind: neuropsychological evidence from a false-belief task with reduced language and executive demands," Cognitive Neuroscience, vol. 16, no. 10, pp. 1773-1784, 2004.

[33] J. D. Henry, L. H. Phillips, J. R. Crawford, M. Ietswaart, and F. Summers, "Theory of mind following traumatic brain injury: The role of emotion recognition and executive dysfunction," Neuropsychologia, vol. 44, no. 10, pp. 1623-1628, 2006.

[34] S. McDonald and S. Pearce, "Clinical insights into pragmatic theory: Frontal lobe deficits and sarcasm," Brain and Language, vol. 53, no. 1, pp. 81-104, 1996.

[35] S. G. Shamay-Tsoory, Y. Tibi-Elhanany, and J. Aharon-Peretz, "The ventromedial prefrontal cortex is involved in understanding affective but not cognitive theory of mind stories," Social Neuroscience, vol. 1, no. 3-4, pp. 149-166, 2006.

[36] C. Williams and R. L. Wood, "Alexithymia and emotional empathy following traumatic brain injury," Journal of Clinical and Experimental Neuropsychology, vol. 32, no. 3, pp. 259-267, 2010.

[37] A. de Sousa, S. McDonald, J. Rushby, S. Li, A. Dimoska, and C. James, "Why don't you feel how I feel? Insight into the absence of empathy after severe Traumatic Brain Injury," Neuropsychologia, vol. 48, no. 12, pp. 3585-3595, 2010.

[38] B. G. Bara, M. Tirassa, and M. Zettin, "Neuropragmatics: neuropsychological constraints on formal theories of dialogue," Brain and Language, vol. 59, no. 1, pp. 7-49, 1997.

[39] F. M. Bosco, A. Parola, K. Sacco, M. Zettin, and R. Angeleri, "Communicative-pragmatic disorders in traumatic brain injury: The role of theory of mind and executive functions," Brain and Language, vol. 168, pp. 73-83, 2017.

[40] S. Channon and S. Crawford, "Mentalising and social problemsolving after brain injury," Neuropsychological Rehabilitation, vol. 20 , no. 5, pp. 739-759, 2010.

[41] S. Channon, A. Pellijeff, and A. Rule, "Social cognition after head injury: sarcasm and theory of mind," Brain and Language, vol. 93, no. 2, pp. 123-134, 2005.

[42] L. M. Grattan and P. J. Eslinger, "Higher cognition and social behavior: Changes in cognitive flexibility and empathy after cerebral lesions," Neuropsychology, vol. 3, no. 3, pp. 175-185, 1989.

[43] R. Griffin, O. Friedman, J. Ween, E. Winner, F. Happé, and H. Brownell, "Theory of mind and the right cerebral hemisphere: Refining the scope of impairment," Laterality, vol. 11, no. 3, pp. 195-225, 2006.

[44] V. Havet-Thomassin, P. Allain, F. Etcharry-Bouyx, and D. Le Gall, "What about theory of mind after severe brain injury?" Brain Injury, vol. 20, no. 1, pp. 83-91, 2006. 
[45] C. A. Honan, S. McDonald, A. Gowland, A. Fisher, and R. K. Randall, "Deficits in comprehension of speech acts after TBI: The role of theory of mind and executive function," Brain and Language, vol. 150, pp. 69-79, 2015.

[46] I. Martin and S. McDonald, "Evaluating the causes of impaired irony comprehension following traumatic brain injury," Aphasiology, vol. 19, no. 8, pp. 712-730, 2005.

[47] I. Martin and S. McDonald, “That Can’t Be Right! What Causes Pragmatic Language Impairment Following Right Hemisphere Damage?" Brain Impairment, vol. 7, no. 03, pp. 202-211, 2006.

[48] S. McDonald and S. Flanagan, "Social perception deficits after traumatic brain injury: Interaction between emotion recognition, mentalizing ability, and social communication," Neuropsychology, vol. 18, no. 3, pp. 572-579, 2004.

[49] S. McDonald, A. Fisher, S. Flanagan, and C. A. Honan, "Impaired perception of sincerity after severe traumatic brain injury," Journal of Neuropsychology, vol. 11, no. 2, pp. 291-304, 2017.

[50] M. Milders, S. Fuchs, and J. R. Crawford, "Neuropsychological impairments and changes in emotional and social behaviour following severe traumatic brain injury," Journal of Clinical and Experimental Neuropsychology, vol. 25, no. 2, pp. 157-172, 2003.

[51] M. Milders, M. Ietswaart, J. R. Crawford, and D. Currie, "Impairments in theory of mind shortly after traumatic brain injury and at 1-year follow-up," Neuropsychology, vol. 20, no. 4, pp. 400-408, 2006.

[52] D. Neumann, B. Zupan, J. F. Malec, and F. Hammond, "Relationships between alexithymia, affect recognition, and empathy after traumatic brain injury," The Journal of Head Trauma Rehabilitation, vol. 29, no. 1, pp. E18-E27, 2014.

[53] P. Njomboro, G. W. Humphreys, and S. Deb, "Exploring social cognition in patients with apathy following acquired brain damage," BMC Neurology, vol. 14, no. 1, article no. 18, 2014.

[54] A. D. Rowe, P. R. Bullock, C. E. Polkey, and R. G. Morris, “'Theory of mind' impairments and their relationship to executive functioning following frontal lobe excisions," Brain, vol. 124, no. 3, pp. 600-616, 2001.

[55] J. M. Spikman, M. E. Timmerman, M. V. Milders, W. S. Veenstra, and J. van der Naalt, "Social cognition impairments in relation to general cognitive deficits, injury severity, and prefrontal lesions in traumatic brain injury patients," Journal of Neurotrauma, vol. 29, no. 1, pp. 101-111, 2012.

[56] L. Turkstra, R. Norman, B. Mutlu, and M. Duff, "Impaired theory of mind in adults with traumatic brain injury: A replication and extension of findings," Neuropsychologia, vol. 111, pp. 117-122, 2018.

[57] S. Baron-Cohen, M. O'Riordan, V. Stone, R. Jones, and K. Plaisted, "Recognition of faux pas by normally developing children and children with asperger syndrome or high-functioning autism," Journal of Autism and Developmental Disorders, vol. 29, no. 5, pp. 407-418, 1999.

[58] S. Baron-Cohen, S. Wheelwright, J. Hill, Y. Raste, and I. Plumb, "The "Reading the Mind in the Eyes" Test revised version: A study with normal adults, and adults with Asperger syndrome or high-functioning autism," Journal of Child Psychology and Psychiatry and Allied Disciplines, vol. 42, no. 2, pp. 241-251, 2001.

[59] S. Channon and S. Crawford, "Problem-solving in real-lifetype situations: The effects of anterior and posterior lesions on performance," Neuropsychologia, vol. 37, no. 7, pp. 757-770, 1999.

[60] H. L. Gallagher, F. Happé, N. Brunswick, P. C. Fletcher, U. Frith, and C. D. Frith, "Reading the mind in cartoons and stories: an
fMRI study of 'theory of mind' in verbal and nonverbal tasks," Neuropsychologia, vol. 38, no. 1, pp. 11-21, 2000.

[61] B. H. Price, K. R. Daffner, R. M. Stowe, and M. M. Mesulam, "The comportmental learning disabilities of early frontal lobe damage," Brain, vol. 113, no. 5, pp. 1383-1393, 1990.

[62] M. D. Lezak, "Living with the characterologically altered brain injured patient," Journal of Clinical Psychiatry, vol. 39, no. 7, pp. 592-598, 1978.

[63] S. McDonald, "Impairments in social cognition following severe traumatic brain injury," Journal of the International Neuropsychological Society, vol. 19, no. 3, pp. 231-246, 2013.

[64] E. Kendall, D. Shum, D. Halson, S. Bunning, and M. Teh, "The Assessment of Social Problem Solving Ability Following Traumatic Brain Injury," The Journal of Head Trauma Rehabilitation, vol. 12, no. 3, pp. 68-78, 1997.

[65] L. S. Turkstra, "Conversation-based assessment of social cognition in adults with traumatic brain injury," Brain Injury, vol. 22, no. 5, pp. 397-409, 2008.

[66] C. A. Hynes, V. E. Stone, and L. A. Kelso, "Social and emotional competence in traumatic brain injury: New and established assessment tools," Social Neuroscience, vol. 6, no. 5-6, pp. 599$614,2011$.

[67] K. D. Cicerone and L. N. Tanenbaum, "Disturbance of social cognition after traumatic orbitofrontal brain injury," Archives of Clinical Neuropsychology, vol. 12, no. 2, pp. 173-188, 1997.

[68] B. G. Bara, I. Cutica, and M. Tirassa, "Neuropragmatics: Extralinguistic communication after closed head injury," Brain and Language, vol. 77, no. 1, pp. 72-94, 2001.

[69] D. N. Brooks and W. McKinlay, "Personality and behavioural change after severe blunt head injury - a relative's view," Journal of Neurology, Neurosurgery \& Psychiatry, vol. 46, no. 4, pp. 336344, 1983.

[70] I. V. Thomsen, "Late outcome of very severe blunt head trauma: A 10-15 year second follow-up," Journal of Neurology, Neurosurgery \& Psychiatry, vol. 47, no. 3, pp. 260-268, 1984.

[71] G. Kinsella, S. Packer, and J. Olver, "Maternal reporting of behaviour following very severe blunt head injury," Journal of Neurology, Neurosurgery \& Psychiatry, vol. 54, no. 5, pp. 422426, 1991.

[72] N. V. Marsh, D. A. Kersel, J. H. Havill, and J. W. Sleigh, "Caregiver burden at 1 year following severe traumatic brain injury," Brain Injury, vol. 12, no. 12, pp. 1045-1059, 1998.

[73] J. Ponsford, J. Olver, M. Ponsford, and R. Nelms, "Long-term adjustment of families following traumatic brain injury where comprehensive rehabilitation has been provided," Brain Injury, vol. 17, no. 6, pp. 453-468, 2003.

[74] R. Wells, J. Dywan, and J. Dumas, "Life satisfaction and distress in family caregivers as related to specific behavioural changes after traumatic brain injury," Brain Injury, vol. 19, no. 13, pp. 1105-1115, 2005.

[75] H. L. Soberg, C. Røe, A. Anke et al., "Health-related quality of life 12 months after severe traumatic brain injury: a prospective nationwide cohort study," Journal of Rehabilitation Medicine, vol. 45, no. 8, pp. 785-791, 2013.

[76] M. Bullinger, P. Azouvi, N. Brooks et al., "Quality of life in patients with traumatic brain injury-basic issues, assessment and recommendations: Results of a consensus meeting," Restorative Neurology and Neuroscience, vol. 20, no. 3-4, pp. 111$124,2002$.

[77] M. Dennis, A. Agostino, C. Roncadin, and H. Levin, "Theory of mind depends on domain-general executive functions of 
working memory and cognitive inhibition in children with traumatic brain injury," Journal of Clinical and Experimental Neuropsychology, vol. 31, no. 7, pp. 835-847, 2009.

[78] P. J. Eslinger and A. R. Damasio, "Severe disturbance of higher cognition after bilateral frontal lobe ablation: Patient EVR," Neurology, vol. 35, no. 12, pp. 1731-1741, 1985.

[79] R. J. R. Blair and L. Cipolotti, "Impaired social response reversal. A case of 'acquired sociopathy,' Brain, vol. 123, no. 6, pp. 11221141, 2000.

[80] D. Tranel, A. Bechara, and N. L. Denburg, "Asymmetric functional roles of right and left ventromedial prefrontal cortices in social conduct, decision-making, and emotional processing," Cortex, vol. 38, no. 4, pp. 589-612, 2002.

[81] J. P. Keenan, J. G. G. Gallup, and D. Falk, The Face in Themirror: The Search for the Origins of Consciousness, HarperCollins Publishers, London, UK, 2003.

[82] G. P. Prigatano and D. L. Schacter, Awareness of Deficit after Brain Injury: Clinical and Theoretical Issues, Oxford University Press, New York, NY, USA, 1991.

[83] Y. Ben-Yishay, J. Rattok, P. Lakin et al., "Neuropsychologic Rehabilitation: Quest for a Holistic Approach," Seminars in Neurology, vol. 5, no. 03, pp. 252-259, 1985.

[84] G. P. Prigatano, D. J. Fordyce, H. K. Zeiner, J. R. Roueche, M. Pepping, and B. C. Wood, Neuropsychological Rehabilitation after Brain Injury, Johns Hopkins University Press, Maryland, Md, USA, 1986.

[85] M. Sherer, T. Hart, and T. G. Nick, "Measurement of impaired self-awareness after traumatic brain injury: A comparison of the patient competency rating scale and the awareness questionnaire," Brain Injury, vol. 17, no. 1, pp. 25-37, 2003.

[86] M. Giustini, E. Longo, E. Azicnuda et al., "Health-related quality of life after traumatic brain injury: Italian validation of the QOLIBRI," Functional Neurology, vol. 29, no. 3, pp. 167-176, 2014.

[87] R. Formisano, E. Longo, E. Azicnuda et al., "Quality of life in persons after traumatic brain injury as self-perceived and as perceived by the caregivers," Neurological Sciences, vol. 38, no. 2, pp. 279-286, 2016.

[88] P. Ciurli, U. Bivona, C. Barba et al., "Metacognitive unawareness correlates with executive function impairment after severe traumatic brain injury," Journal of the International Neuropsychological Society, vol. 16, no. 2, pp. 360-368, 2010.

[89] V. E. Stone, S. Baron-Cohen, and R. T. Knight, "Frontal lobe contributions to theory of mind," Cognitive Neuroscience, vol. 10, no. 5, pp. 640-656, 1998.

[90] M. D. Lezak, Neuropsychological Assessment, Oxford University Press, Oxford, UK, 1995.

[91] S. Schwartz, J. Baldo, R. E. Graves, and P. Brugger, "Pervasive influence of semantics in letter and category fluency: a multidimensional approach," Brain and Language, vol. 87, no. 3, pp. 400-411, 2003.

[92] T. Shallice, "Specific impairments of planning," in The neuropsychology of cognitive functions, D. E. Broadbent and L. Weiskrantz, Eds., pp. 199-209, The Royal Society, London, UK, 1982.

[93] J. R. Stroop, "Studies of interference in serial verbal reactions," Journal of Experimental Psychology, vol. 18, no. 6, pp. 643-662, 1935.

[94] H. E. Nelson, "A modified card sorting test sensitive to frontal lobe defects," Cortex, vol. 12, no. 4, pp. 313-324, 1976.
[95] R. M. Reitan, "Validity of the trail making test as an indicator of organic brain damage," Perceptual and Motor Skills, vol. 8, no. 3, pp. 271-276, 1958.

[96] A. Mehrabian, Manual for the Balanced Emotional Empathy Scale (BEES), 2000, Albert Mehrabian, Monterey, CA.

[97] R. M. Bagby, J. D. A. Parker, and G. J. Taylor, "The twentyitem Toronto Alexithymia scale-I. Item selection and crossvalidation of the factor structure," Journal of Psychosomatic Research, vol. 38, no. 1, pp. 23-32, 1994.

[98] D. Kahneman and A. Tversky, "he simulation heuristic," in Judgment under Uncertainty: Heuristics and Biases, D. Kahneman, P. Slovic, and A. Tversky, Eds., pp. 201-210, Cambridge University Press, New York, NY, USA, 1982.

[99] B. Jennett, "Defining brain damage after head injury," Journal of the Royal College of Physicians, vol. 13, no. 4, pp. 197-200, 1979.

[100] C. Long and L. Ross, Handbook of Head Trauma. Acute Care of Recovery, vol. 8, Plenum Press, New York, NY, USA, 1992.

[101] S. McDonald, L. Togher, and C. Code, "Communication disorders following traumatic brain injury," The Journal of Head Trauma Rehabilitation, vol. 16, no. 6, pp. 612-619, 1999.

[102] U. Bivona, P. Ciurli, C. Barba et al., "Executive function and metacognitive self-awareness after Severe Traumatic Brain Injury," Journal of the International Neuropsychological Society, vol. 14, no. 5, pp. 862-868, 2008.

[103] B. Crosson, P. P. Barco, C. A. Velozo et al., "Awareness and compensation in postacute head injury rehabilitation," The Journal of Head Trauma Rehabilitation, vol. 4, no. 3, pp. 46-54, 1989.

[104] M. H. Davis, "Measuring individual differences in empathy: Evidence for a multidimensional approach," Journal of Personality and Social Psychology, vol. 44, no. 1, pp. 113-126, 1983.

[105] S. Baron-Cohen and S. Wheelwright, "The empathy quotient: an investigation of adults with asperger syndrome or high functioning autism, and normal sex differences," Journal of Autism and Developmental Disorders, vol. 34, no. 2, pp. 163-175, 2004. 




The Scientific World Journal
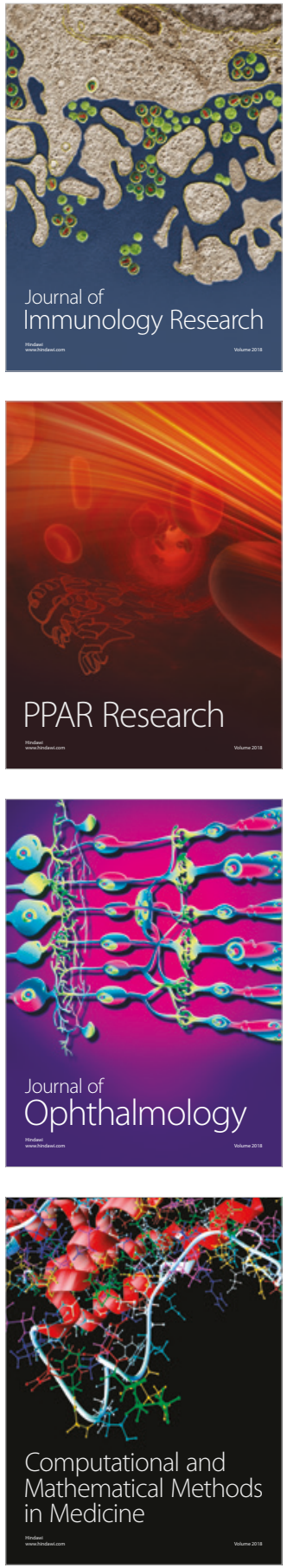

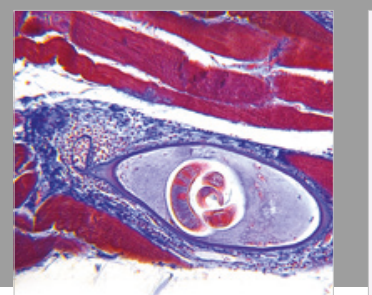

Gastroenterology Research and Practice



\section{Hindawi}

Submit your manuscripts at

www.hindawi.com
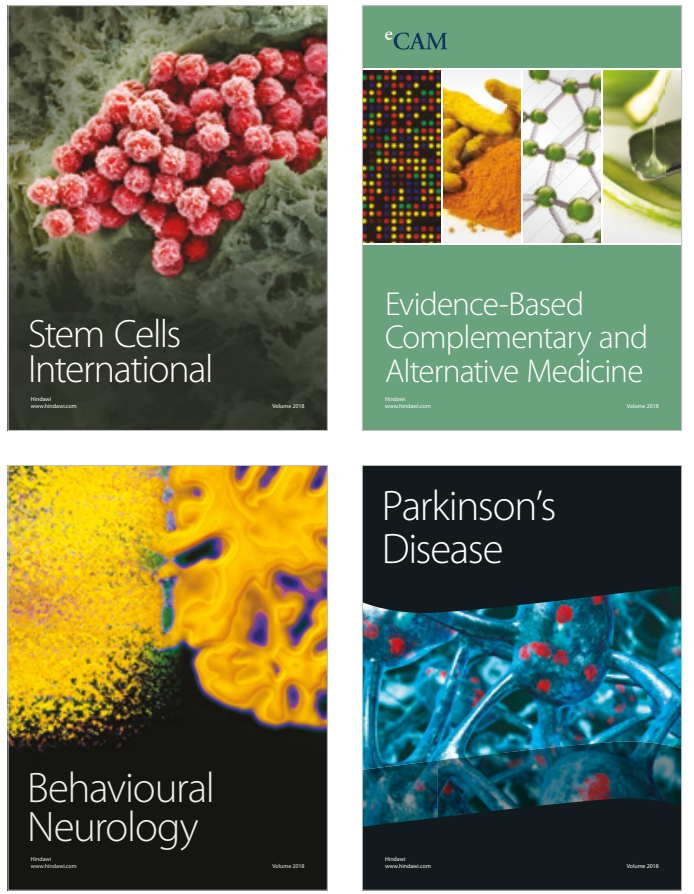



ournal of

Diabetes Research

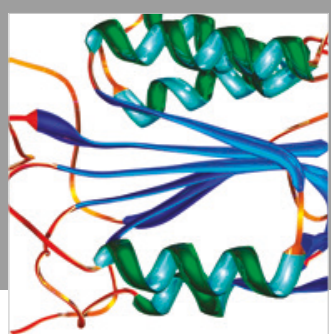

Disease Markers
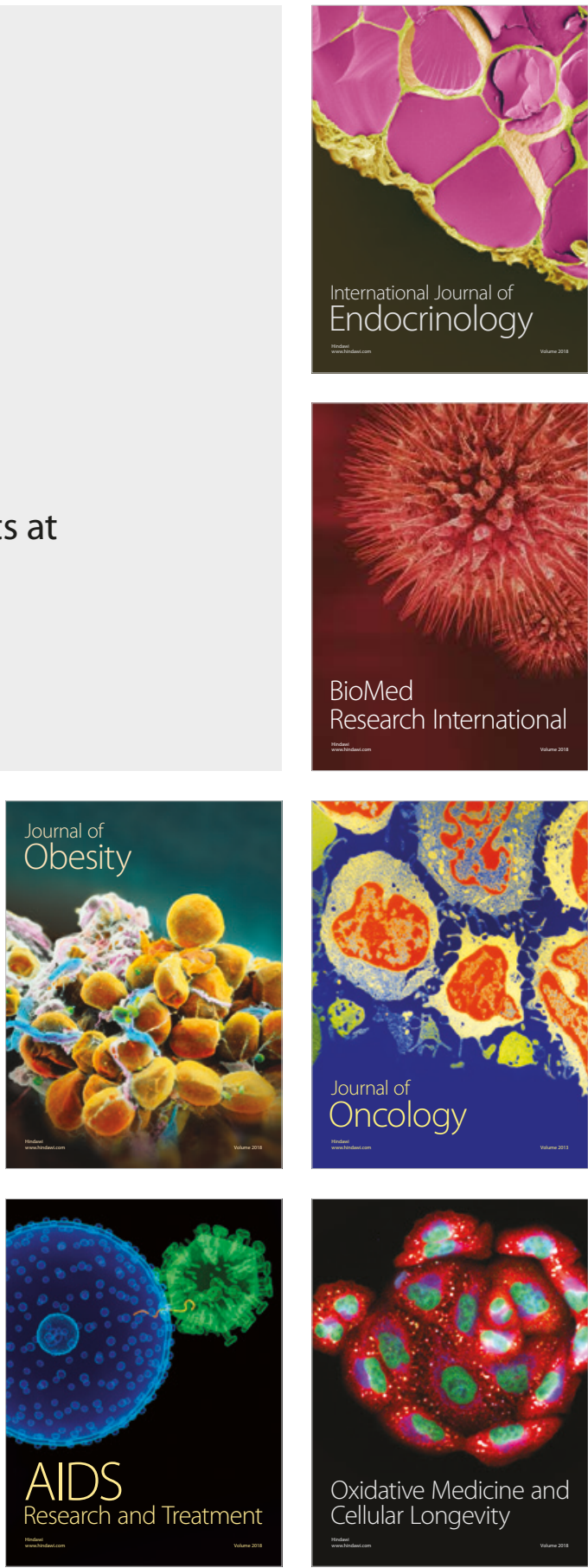